\title{
microRNA-330 inhibits cell motility by downregulating Sp1 in prostate cancer cells
}

\author{
YEQING MAO, HONG CHEN, YIWEI LIN, XIN XU, ZHENGHUI HU, \\ YI ZHU, JIAN WU, XIANGLAI XU, XIANGYI ZHENG and LIPING XIE \\ Department of Urology, The First Affiliated Hospital, School of Medicine, \\ Zhejiang University, Hangzhou, Zhejiang 310003, P.R. China
}

Received January 10, 2013; Accepted April 16, 2013

DOI: $10.3892 /$ or.2013.2452

\begin{abstract}
RNAs (miRNAs), small non-coding RNAs, have emerged as key regulators of a large number of genes. The present study aimed to explore novel biological functions of miR-330 in the human prostate cancer cell lines DU145 and PC3. We confirmed that miR-330 was downregulated and inversely correlated with specificity protein 1 (Sp1) expression. Overexpression of miR-330 by transfection of a chemically synthesized miR-330 mimic induced a reduction in expression levels of the $\mathrm{Sp} 1$ protein, accompanied by significant suppression of cellular migration and invasion capability. In addition, the Spl-knockdown experiments presented similar phenomena. Finally, the luciferase reporter assay validated $\mathrm{Sp} 1$ as the direct target of miR-330. These findings indicate that miR-330 acts as an anti-metastatic miRNA in prostate cancer.
\end{abstract}

\section{Introduction}

microRNAs (miRNAs) are recognized as a class of small (19-25 nt) non-coding RNAs that play important roles in gene regulation by partially or fully complementary matching with the 3'-untranslated region (UTR) of target mRNAs, and trigger a transcriptional and/or post-transcriptional suppression of the target gene $(1,2)$. They are involved in numerous physiological functions such as cell differentiation, migration, proliferation, apoptosis and senescence (3). Recently, it was reported that more than 1,000 human miRNAs frequently target regions related to cancer development (4), and this complex collection could be further classified as oncogenic, tumor-suppressive, or context-dependent miRNAs (5).

Correspondence to: Dr Liping Xie, Department of Urology, The First Affiliated Hospital, School of Medicine, Zhejiang University, Qinchun Road 79, Hangzhou, Zhejiang 31003, P.R. China

E-mail: xielp@zjuem.zju.edu.cn

Key words: microRNA-330, prostate cancer, migration, invasion, specificity protein 1
miR-330 was first discovered by Weber (6), and described as a downregulated miRNA in tumors by Gaur et al (7), when the authors compared the differential expression patterns of 241 miRNAs between 13 normal and 59 tumor-derived cell lines. This finding was further confirmed by Ruike et al (8) who investigated the miRNA and mRNA expression associations across 16 human cell lines. mir-330 is located on chromosome $19 \mathrm{q} 13$, a fragile point which was reportedly associated with tumor aggressiveness in prostate cancer $(9,10)$. To date, investigations concerning the biological functions of miR-330 in cancer are sparse. Recently, Qu et al (11) reported its oncogenic role in human glioblastoma by regulating the SH3GL2 gene. In prostate cancer, however, it was reported as a tumor-suppressor by Lee et al (12), who demonstrated its significantly lower expression and inverse correlation with E2F1 in both prostate cancer cell lines and cancer specimens. They further discovered its proapoptotic role through E2F1-mediated suppression of Akt phosphorylation.

Specificity protein 1 (Sp1), the essential member of the $\mathrm{Sp} / \mathrm{Krupel}-\mathrm{like}$ (KLF) transcription factor family, is regarded as ubiquitous in cells and participates in the tumorigenesis of a variety of cancers including prostate (13). It plays an important role in tumor progression, including cell proliferation, angiogenesis, differentiation, apoptosis, migration and invasion (14), representing itself as an ideal target for cancer treatment. Here, we report the post-transcriptional regulation of Sp1 by miR-330. Our investigation elucidated the tumor-suppressive role of miR-330 in prostate cancer in an anti-migratory and -invasive manner.

\section{Materials and methods}

Reagents. All the RNA duplexes were synthesized by GenePharma Co., Ltd. (Shanghai China). The sense sequences of the duplexes were as follows: miR-330a mimic (termed as miR-330), 5'-GCAAAGCACACGGCCUGCAGAGA-3'; negative control RNA (termed as NC), 5'-ACUACUGAGUGACA GUAGA-3'; small interfering RNA for Sp1 (termed as siRNA), 5'-AAAGCGCUUCAUGAGGAGUGA-3' (15). All of the primers for quantitative real-time PCR (qPCR) were synthesized by Sangon Biotech (Shanghai China), whose sequences were as follows: GAPDH forward, 5'-AAGGTGAAGGTCG GAGTCA-3' and reverse, 5'-GGAAGATGGTGATGGGATT 
T-3'; Sp1 forward, 5'-AGTTCCAGACCGTTGATGGG-3' and reverse, 5'-GTTTGCACCTGGTATGATCTGT-3'; matrix metalloproteinase-2 (MMP2) forward, 5'-TACAGGATCATT GGCTACACACC-3' and reverse, 5'-GGTCACATCGCTCC AGACT-3'; MMP9 forward, 5'-TGTACCGCTATGGTTAC ACTCG-3' and reverse, 5'-GGCAGGGACAGTTGCTTCT-3'. The primary antibodies were, anti-GAPDH (Epitomics, Burlingame, CA, USA), anti-Sp1, anti-MMP2 (both from Santa-Cruz Biotechnology, Santa Cruz, CA, USA) and antiMMP9 (Epitomics).

Cell culture and transfection. The human prostate cancer cell lines DU145 and PC3 and the normal prostate epithelial cell line RWPE were purchased from Shanghai Institute of Cell Biology, Chinese Academy of Sciences. Cells were cultured in RPMI-1640 medium supplemented with $10 \%$ heat-inactivated fetal bovine serum (FBS) (Gibco-BRL, Grand Island, NY, USA), penicillin $(50 \mathrm{U} / \mathrm{ml})$, and streptomycin $(50 \mu \mathrm{g} / \mathrm{ml})$ in a humidified atmosphere containing $5 \% \mathrm{CO}_{2}$ maintained at $37^{\circ} \mathrm{C}$. For miR-330 overexpression, we chose miR-330a mimic transfection, which is efficient and economical. Briefly, cells were seeded in a 6-well plate at a density of 20-40x $10^{4}$ cells/well in medium without antibiotics. At $\sim 60-70 \%$ confluency, transfection was performed using Lipofectamine ${ }^{\mathrm{TM}} 2000$ reagent purchased from Invitrogen Life Technologies (Carlsbad, CA, USA) according to the manufacturer's instructions. The transfection efficiency was assessed by qPCR.

$R N A$ isolation and $q P C R$. Cells were harvested $48 \mathrm{~h}$ after transfection, and were treated with RNAiso for small RNA and RNAiso Plus for total RNA. The extracted small RNA and total RNA were transcribed into cDNA with the One Step PrimeScript miRNA cDNA Synthesis kit and PrimeScript RT reagent kit (Takara, Japan), respectively. For quantification, qPCR was performed using SYBR Premix Ex Taq (Takara) with the 7500 Fast Sequence Detection System (Applied Biosystems, Foster City, CA, USA). After the Ct values of the target genes were subtracted by that of the internal control GAPDH, the relative expression level of each target gene was obtained using the $2^{-\Delta \Delta \mathrm{Ct}}$ method.

Apoptosis assay. Apoptosis was assessed by the Annexin V-FITC kit (Invitrogen Life Technologies). Both cell lines were transfected for $48 \mathrm{~h}$, and were harvested and stained with Annexin V-FITC and PI according to the manufacturer's instructions. Cell samples were analyzed by BD LSR II Flow Cytometry System with FACSDiva software (BD Biosciences, Franklin Lakes, NJ, USA) and apoptotic cell fractions were determined.

Cell cycle analysis. Briefly, cells were harvested $48 \mathrm{~h}$ after transfection, washed with phosphate-buffered saline (PBS), detrypsinized, resuspended and finally fixed in $75 \%$ ethanol at $-20^{\circ} \mathrm{C}$ for $24 \mathrm{~h}$ after removal of the supernatant. Subsequently, cells were washed with PBS, and incubated with DNA Prep Stain (Beckman Coulter, Fullerton, CA, USA) for $30 \mathrm{~min}$. We used the BD LSR II Flow Cytometry System with FACSDiva software to detect cell cycle distribution, which was analyzed by ModFit LT software package and presented as the percentage of cell counts in every cycle for the G1, S and G2 phases.
Western blot analysis. Cells were harvested $\sim 48 \mathrm{~h}$ after treatment, and lysed in RIPA buffer; the isolated protein was quantified using the Bradford protein assay (Bio-Rad, Hercules, CA, USA) and separated with equivalent loading on $10 \%$ SDS-polyacrylamide gels subsequently transferred to nitrocellulose membranes (Millipore, Bedford, MA, USA). The membranes were blocked with 5\% non-fat milk and incubated with the primary antibody with the recommended dilution rate at $4^{\circ} \mathrm{C}$ for at least $12 \mathrm{~h}$, followed by incubation with the corresponding horseradish peroxidase (HRP)-conjugated secondary antibody at an appropriate dilution in TBS-Tween at room temperature for $1 \mathrm{~h}$. Finally, Enhanced Chemiluminescence Plus reagent (Millipore) was used to detect the bound secondary antibody.

Transwell assay. Millicell Transwell inserts (8- $\mu \mathrm{m})$ (Millipore) were placed in a 24-well plate (Corning Incorporated, Corning, NY, USA) with $600 \mu$ l serum-free RPMI-1640 medium in the lower chamber. The oligo-treated cells were harvested, counted and seeded (6-8x10 cells/well) to the upper chamber. For the invasion assay, the wells were coated with Matrigel (BD Biosciences, Canada) diluted with serum-free RPMI1640 medium in a 1:8 ratio before seeding. After incubation at $37^{\circ} \mathrm{C}$ with $5 \% \mathrm{CO}_{2}$ for $48 \mathrm{~h}$, the inserts were withdrawn and fixed in cold methanol for $10 \mathrm{~min}$ and then stained with $0.5 \%$ crystal violet in PBS. After 15 min of incubation, the inserts were washed thoroughly in water and the cells on the upper surfaces of the membranes were removed, and then dried overnight. The membranes were excised and fixed on slides with neutral resin, and the cells fixed to the bottom were observed using an inverted microscope.

Plasmid construction and luciferase reporter assay. For investigating the possible target genes of miR-330, we used the target prediction program Targetscan (http://www. targetscan.org/). As the program predicted, the potential target gene Sp1 has 2 putative binding sites in its 3'-UTR. Therefore, we designed 2 pairs of primers for amplification from the cDNA of RWPE cells: Site 1 forward, 5'-TGCAAGGTAGCATGGGTCCAAGAG-3' and reverse, 5'-TGGTGGGAAGCCAAGACAACA-3'; Site 2 forward, 5'-GTCGCAGCAGTAGCTTTGGGGA-3' and reverse, 5'-ACTTAGGGCAGTTGAGAGGCAGA-3'. The products were then inserted into the pmirGLO Dual-Luciferase miRNA target expression vector (Promega Corporation, Madison, WI, USA) between the SacI and SalI sites (named pGL-WT1 and pGL-WT2). All the insertions were confirmed by sequencing. HEK293T cells were plated in a 24 -well plate and cultured for $24 \mathrm{~h}$. Co-transfection was performed with $50 \mathrm{nM}$ of either the miR-330 mimic or NC oligo and $100 \mathrm{ng}$ wild-type reporter plasmid. The relative luciferase activity was measured by the Dual-Luciferase reporter assay system (Promega) $24 \mathrm{~h}$ after transfection.

Statistical analysis. All the graphical data were constructed by GraphPad Prism version 5 for Windows. Data were analyzed by use of SPSS 15.0 software, and are expressed as means \pm standard deviation (SD) for each group. The $t$-test or one-way ANOVA was used to make comparisons; $\mathrm{P}<0.05$ was considered to indicate a statistically significant result. 


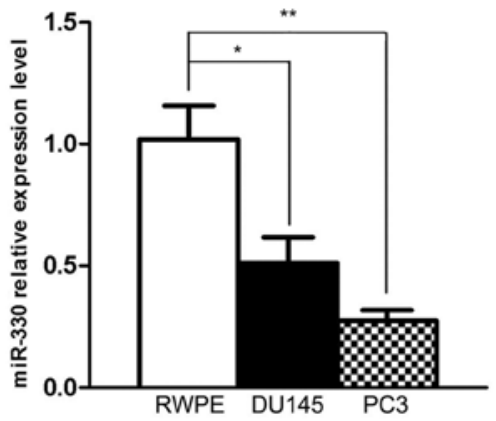

Figure 1. Quantification of miR-330 in prostate cancer cell lines. The relative expression level of miR-330 was decreased by 0.5 -fold in the DU145 cells and 0.3 -fold in PC3 cells when compared with miR-330 in the normal epithelial cell line.

\section{Results}

miR-330 is downregulated in prostate cancer cells. To confirm the expression levels of miR-330 in the human prostate cancer cell lines, mRNAs were extracted from DU145, PC3 and RWPE cells, and then transcribed into the template for qPCR quantification analysis. We used U6 RNA level as an internal control. Compared with the RWPE cells, both cancer cell lines exhibited significantly decreased expression of miR-330 when normalized to U6 [DU145 downregulated by 0.5 -fold $(\mathrm{P}<0.05)$, and $\mathrm{PC} 3$ by 0.7 -fold $(\mathrm{P}<0.01)]$ (Fig. 1). This result indicates that miR-330 is downregulated in prostate cancer cells.

Cell migration is inhibited by miR-330 in prostate cancer. To determine whether miR-330 affects cell migratory capacity, cells with miR-330 overexpression were subjected to a Transwell assay (FBS-induced migration and Matrigel invasion test). After 24 to $48 \mathrm{~h}$, the migrating cells were fixed, stained and observed microscopically. As the representative micrographs clearly demonstrate, miR-330 overexpression led to potent inhibition of cell migration and invasion (Fig. 2).

Neither apoptosis nor cell cycle arrest is induced by miR-330 in prostate cancer cells. To investigate the biological function of miR-330 we performed flow cytometry, as previous reports indicate a pro-apoptotic role of miR-330. Both cell lines were treated by either miR-330 or NC for $48 \mathrm{~h}$, and flow cytometric analysis was carried out. There was an insignificant difference in the percentage of apoptotic cells between the miR-330 and NC pretreatment groups (DU145, $7.37 \pm 2.65$ vs. $5.87 \pm 1.95$; $\mathrm{P}>0.05$ and $\mathrm{PC} 3,5.47 \pm 1.48$ vs. $6.70 \pm 2.09 ; \mathrm{P}>0.05$ ) (Fig. $3 \mathrm{~A}$ ). Likewise, cell cycle analysis did not reveal any obvious change in cell cycle distribution in G1, S, G2 phases between both cell groups ( $\mathrm{P}>0.05)$ (Fig. 3B). Therefore, miR-330 did not affect apoptosis or cell cycle arrest in the prostate cancer cells, at least during our observation period.

Sp1 is post-transcriptionally downregulated by miR-330. To further investigate the underlying mechanism of miR-330-induced metastatic inhibition, Sp1 was selected for qPCR and western blot analysis, by the aid of a bioinformatic tool. The result showed that following miR-330 overexpression, Sp1 expression was inhibited only at the protein level rather than at the mRNA level, which suggests a post-transcriptional regulation. Expression of other metastasis-related molecules such as MMP2 and MMP9 was also markedly downregulated at the mRNA and protein levels (Fig. 4A) $(\mathrm{P}<0.05)$, which verified the involvement of miR-330 in the regulation of metastasis.

The basic expression levels of Sp1 were also examined and compared between the DU145 and PC3 cells and normal epithelial RWPE cells. Sp1 expression was obviously elevated in the cancer cells and was $\sim 2$-fold increased when compared with the level in the RWPE cells $(\mathrm{P}<0.05)$ (Fig 4B). Considering the above data and the inverse correlation with miR-330 (Fig. 1), we surmised that $\mathrm{Sp} 1$ is very likely the target of miR-330.

Inhibition of cell motility by miP-330 is associated with downregulation of $\mathrm{Sp1}$. For further investigation of the possible novel target Sp1, knockdown technique was employed to evaluate its biological function in vitro. A small interfering RNA (siSp1) designed to knock down the Sp1 gene was used to treat the cancer cells compared with NC. The Transwell assay clearly demonstrated that Sp1 knockdown caused significant suppression of the migratory and invasive capability in much
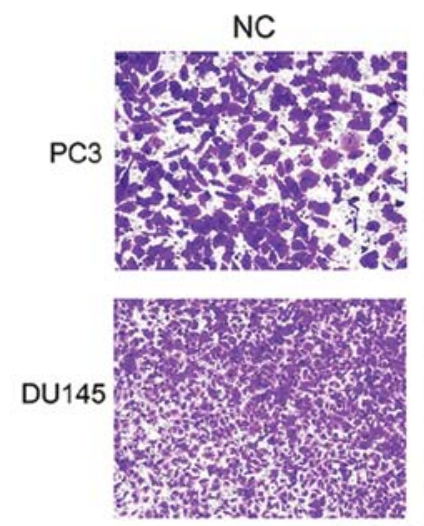

invasion (100x)

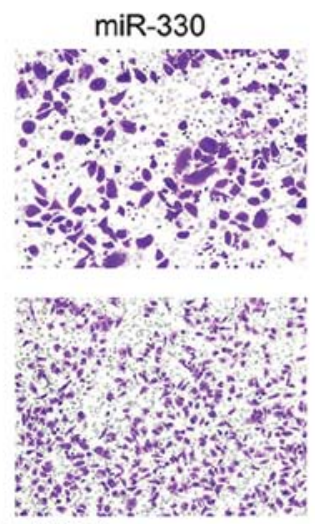

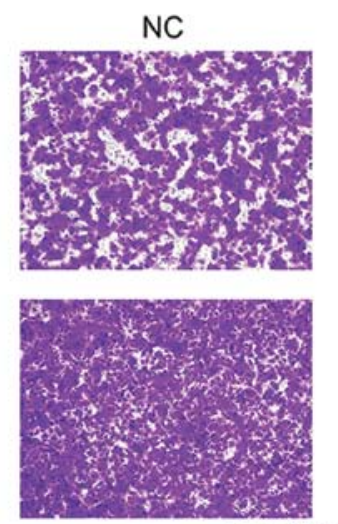
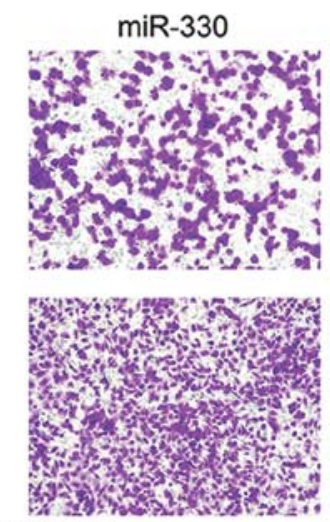

migration (100x)

Figure 2. Cell motility was inhibited by miR-330 in prostate cancer. The representative micrographs (magnification, x100) clearly showed the potent influence of miR-330 on cell invasion and migration capability. 
A
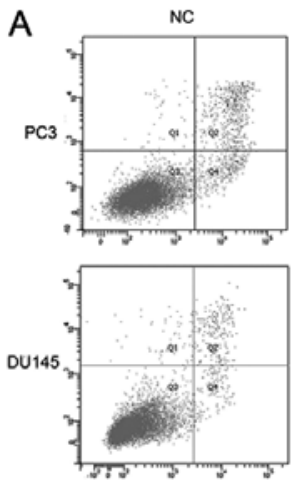
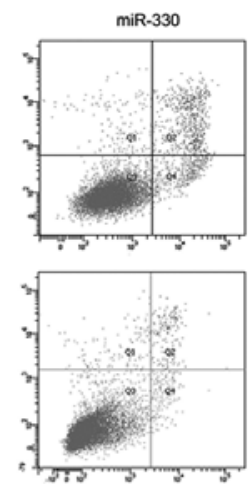
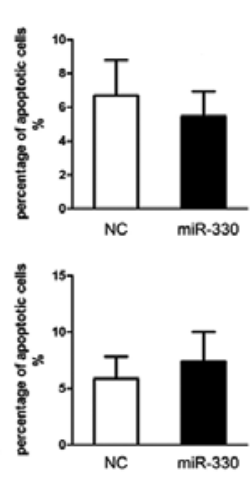

B
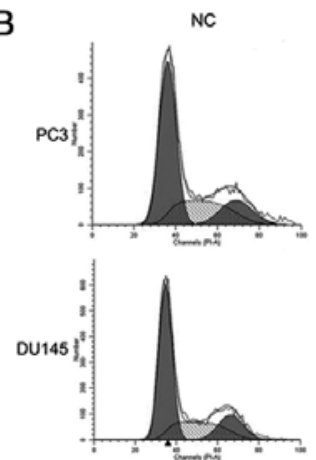
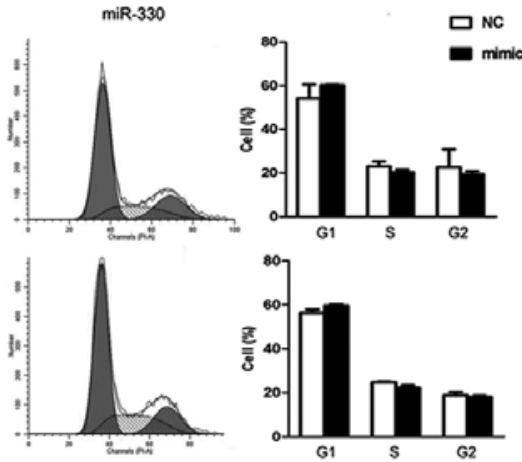

Figure 3. Neither apoptosis nor cell cycle arrest is induced by miR-330 in prostate cancer cells. Apoptosis and cell cycle distribution of DU145 and PC3 cells were examined by flow cytometry $48 \mathrm{~h}$ after transfection. (A) Quadrant (Q)4 represents early apoptotic cells (Annexin $\mathrm{V}^{+} / \mathrm{PI} 2$ ), and Q2 late apoptotic cells (Annexin $\mathrm{V}^{+} / \mathrm{PI}^{+}$); the apoptotic cell population was calculated by the sum of these two percentages, and represented as the means $\pm \mathrm{SD}(\mathrm{P}>0.05)$. (B) The cell populations at the G1, S, G2 phases were indicated by the representative histograms. There was a similar cellular distribution at each cell cycle phase between the $\mathrm{NC}$ group and the mimic group $(\mathrm{P}>0.05)$.

A

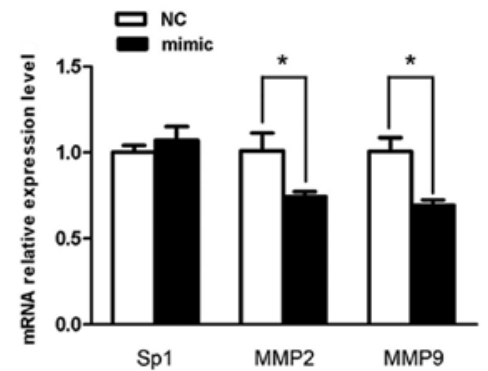

DU145

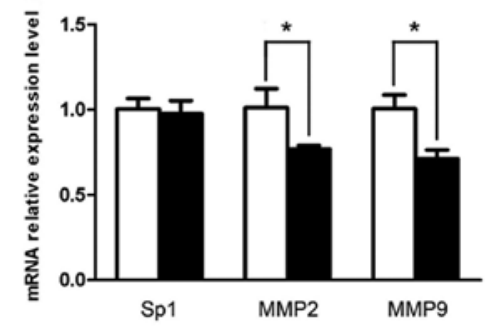

B

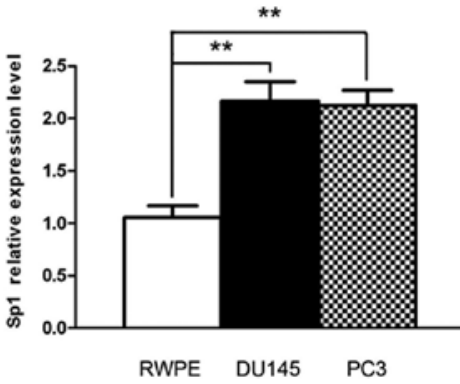

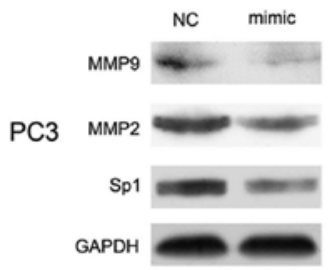
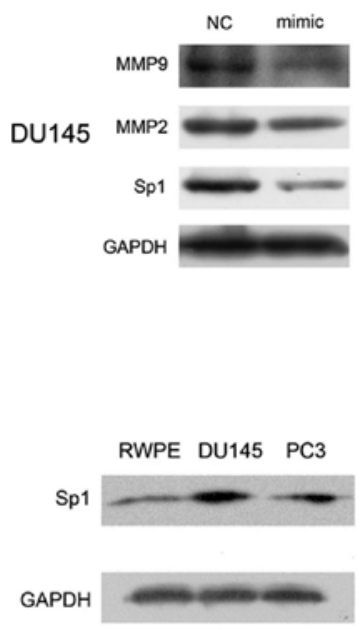

Figure 4. miR-330 post-transcriptionally downregulates specificity protein 1 (Sp1) and is inversely correlated with it. (A) Marked reduction in MMP2 and MMP9 mRNA expression was observed in both prostate cancer cell lines $\left({ }^{*} \mathrm{P}<0.05 ;{ }^{* *} \mathrm{P}<0.01\right)$, while Sp1 was nearly unaffected (P>0.05). Western blotting in parallel indicated the prominent decrease in Sp1, MMP2 and MMP9 at the protein level. (B) An elevated expression level of Sp1 was found in the prostate cancer cell lines compared with that in the RPWE cells $\left({ }^{*} \mathrm{P}<0.05 ;{ }^{* *} \mathrm{P}<0.01\right)$.

the same pattern as miR-330 overexpression (Fig. 5B). In the mechanistic study, Sp1 knockdown, as anticipated, had a similar effect as miR-330 overexpression; MMP2 and MMP9 expression was prominently decreased at both the mRNA and protein levels; a drastic decrease in $\mathrm{Sp1}$ expression indicated efficiency of knockdown (Fig. 5A).
Sp1 is a novel target of $m i R-330$. We further determined the relationship between miR-330 and Sp1. As the bioinformatic tool revealed, two putative target sites were identified, which starts from the 231 base position and the 4035 base position on the 3'UTR sequence respectively (Fig. 6A). Two 400 bp-long fragments around these target sites were cloned downstream 
A
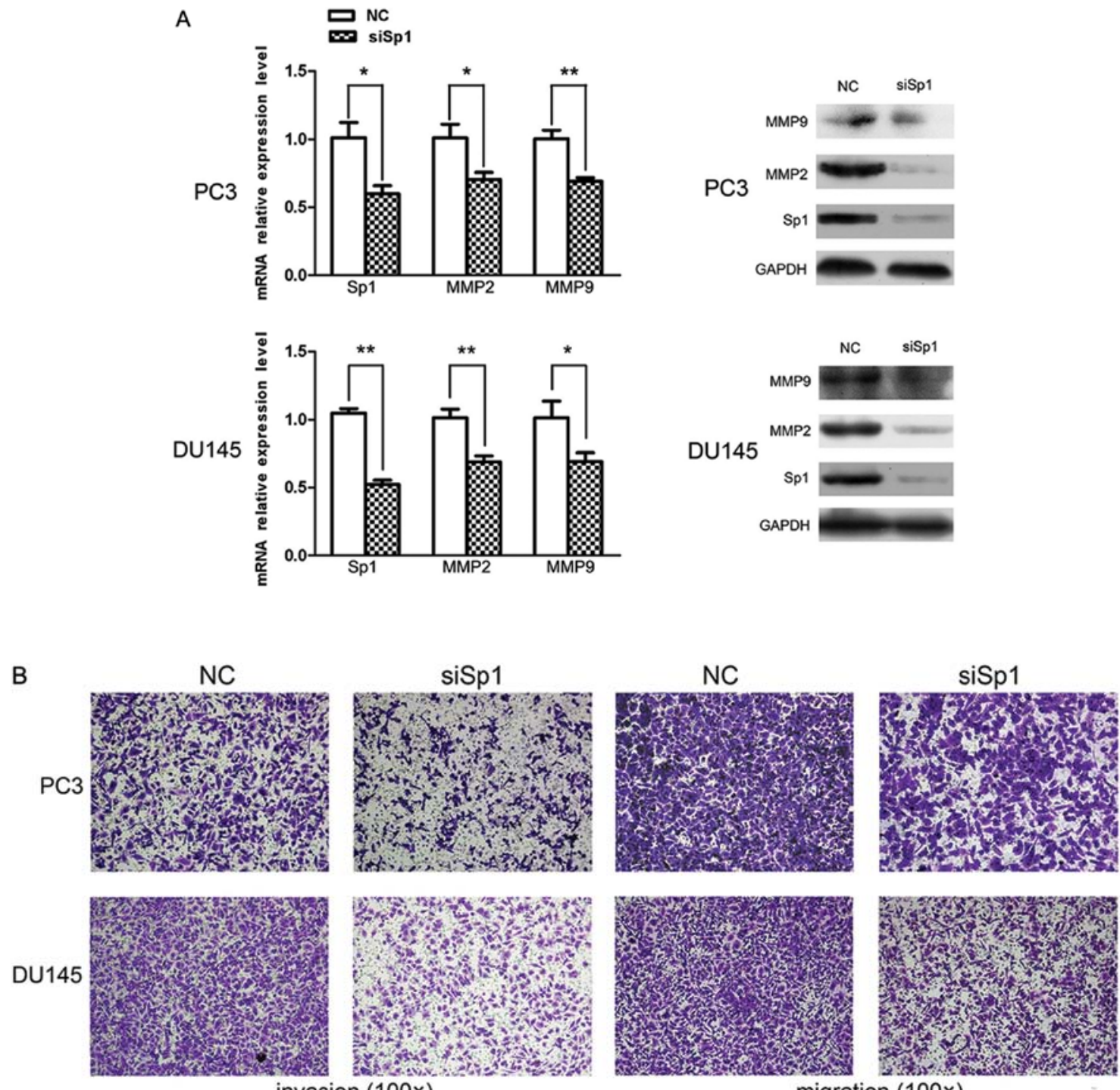

migration $(100 \times)$

Figure 5. Knockdown of specificity protein 1 (Sp1) mimics miR-330 overexpression. (A) qPCR and western blotting showed a significant decrease in Sp1, MMP2 and MMP9 in both prostate cancer cell lines $\left({ }^{*} \mathrm{P}<0.05 ;{ }^{* *} \mathrm{P}<0.01\right)$ after knockdown of Sp1. (B) Representative micrographs (magnification, $\left.\mathrm{x} 100\right)$ of the Transwell assay showed notable impairment of migration and invasion capacity of the cancer cells by Spl knockdown.

of the firefly luciferase of pmirGLO Dual-Luciferase miRNA target expression vector, and termed pGL-WT1 and pGL-WT2, respectively. An individual plasmid with either miR-330 or the NC duplex was used to co-transfect HEK293T cells. The luciferase activity in the groups treated with miR-330 was deceased, when compared with the control; the combination of pGL-WT1 and miR-330 resulted in a more prominent change with the activity value $\sim 40 \%(\mathrm{P}<0.01)$ of the $\mathrm{NC}$ group than pGL-WT2 $(\mathrm{P}<0.05)$ (Fig. 6B). These findings indicate that miR-330 inhibited Sp1 expression through direct binding of the 3'-UTR of its transcript.

\section{Discussion}

As a major health concern among males, prostate cancer is currently the second leading cause of cancer-related death in Western countries. Although reports of improved survival rates of localized or organ-confined tumors are increasingly common, confusion still lies in the problem of dealing with metastasis. Recent studies of metastasis-associated miRNAs have provided us with more insights into cancer pathogenesis $(16,17)$. Watahiki et al (18) compared the miRNA profiling of a transplantable metastatic vs. a non-metastatic prostate cancer xenograft, and discovered 53 pro-metastatic miRNAs and 68 anti-metastatic miRNAs, some of which have been widely accepted as metastic miRs including miR-21, miR-331-3p, miR-205 and miR-203.

miR-330, a recently identified miRNA, has been rarely investigated to date. Although it has been regarded as a downregulated gene in many types of cancer cells, the biological functions are reportedly diverse and contradictory $(11,12)$. Here, we first reported the anti-migratory and anti-invasive role of miR-330 in prostate cancer. The relatively lower expression level of miR-330 in the cancer cell lines PC3 and DU145 when compared with the normal epithelial cell line RWPE was consistent with Lee et al (12). For forced expression, we used miR-330 mimics, the analog of miR-330, via transfectant Lipofectamine 2000 for the sake of accessibility, 

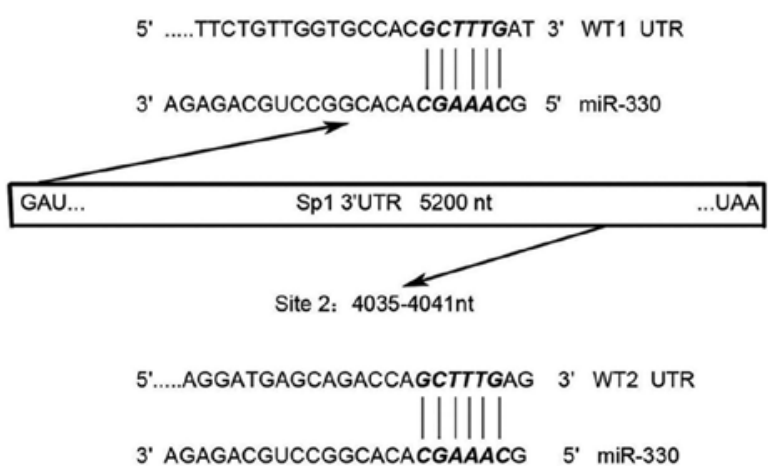

B

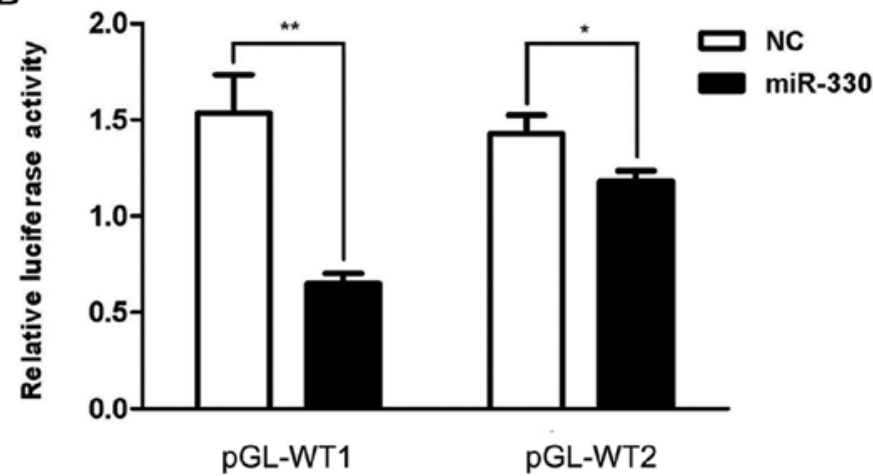

Figure 6. Specificity protein 1 (Spl) is a novel target of miR-330. (A) The schematic illustration represents two potential miR-330-targeted sites on 3 ' UTR of the Sp1 transcript, as predicted by the online bioinformatic tool Targetscan (http://www.targetscan.org/). The '231-237' and '4035-4041' sites are denoted as 'Site 1' and 'Site 2', respectively. (B) HEK293T cells were co-transfected with $50 \mathrm{nmol}$ of either miR-330 or NC and $100 \mathrm{ng}$ of pGL-WT1 or pGL-WT2, and $24 \mathrm{~h}$ later, cells were lysed and assessed for luciferase activity. The relative activity was represented as normalization of the firefly value to the Renilla value and expressed as means $\pm \mathrm{SD}$.

and $\mathrm{qPCR}$ validated the high efficiency of transfection which favorably persisted for 4-5 days (data not shown). As for the functional study, the anti-migration and invasion capacity of miR-330 was clearly demonstrated by the Transwell assay, while its association with apoptosis or cell cycle arrest was not observed in our study. For further mechanistic investigation, we screened Sp1 as the metastasis-associated gene possibly targeted by miR-330, based upon in silico analysis and the inverse relationship between them. qPCR and western blotting revealed post-transcriptional inhibition of Sp1 and a significant decrease in downstream genes MMP2 and MMP9 by miR-330 overexpression; these phenomena were mimicked by $\mathrm{Sp} 1$ knockdown, which further strengthened the hypothesis that miR-330 plays an anti-migratory and -invasive role via $\mathrm{Sp} 1$ in prostate cancer. Finally, the luciferase reporter assay was performed to determined Sp1 as the target gene, and even pinpoint the pairing site ' $231-237$ ' in the Sp1 3'UTR as the major target region.

The implication of Sp proteins in tumorigenesis has been widely accepted, based on the increasing number of reports revealing ubiquitous overexpression of these transcription factors in various neoplastic tissues (19-22). Sp1 regulates thousands of genes including oncogenes and tumor-suppressor genes, via its direct interaction with the components of the basal transcription machinery, TAF4, TAF7 and the recruit- ment of TBP/TFIID (23-26). In prostate cancer, Sp1 was reported to regulate key genes including the androgen receptor (AR), c-Met, FAS, FLIP and TGF. MMP2 and MMP9 are two intensively studied members of the extracellular matrixdegrading gelatinase family, whose regulation reportedly depends on Spl via interaction of their GC-rich sequences in promoters with specific domains of Sp1 (27-29). Here, we confirmed the pivotal role of Sp1 in cell migration and invasion, and demonstrated its close relationship with these matrix metalloproteinases.

It should be noted that Lee et al (12) reported the antiproliferative and pro-apoptotic role of miR-330 in prostate cancer cells through MTT and plate colony formation assays. We performed an apoptosis assay for fear that proliferationrelated factors may interfere with our invasion assay. As the results indicated, both apoptosis and cell cycle analyses yielded an insignificant difference within our observation period. Considering the complex signaling network of miRNAs, we proposed that the pro-apoptotic and anti-proliferative roles of miR-330 may manifest themselves in a latent and moderate manner.

In summary, we identified miR-330 as an anti-metastatic miRNA in prostate cancer. By targeting the 3'UTR of Sp1, miR-330 post-transcriptionally suppressed the expression of Spl protein, and further decreased the levels of MMPs, and 
finally inhibited migration and invasion of prostate cancer cells. Admittedly, our conclusions were derived solely from an in vitro study. Thus, our findings require further confirmation using clinical specimens and in vivo experiments. Yet, they suggest that miR-330 may be a therapeutic target for prostate cancer metastasis.

\section{Acknowledgements}

This study was supported by a grant from the Zhejiang Provincial Natural Science Foundation of China (LY12H05006), the National Key Clinical Speciality Construction Project of China, the Key Medical Disciplines of Zhejiang Province and Zhejiang Provincial Natural Science Foundation of China (Z2090356).

\section{References}

1. Ambros $\mathrm{V}$ and Chen $\mathrm{X}$ : The regulation of genes and genomes by small RNAs. Development 134: 1635-1641, 2007.

2. Hutvágner G and Zamore PD: A microRNA in a multipleturnover RNAi enzyme complex. Science 297: 2056-2060, 2002.

3. Hwang HW and Mendell JT: microRNAs in cell proliferation, cell death, and tumorigenesis. Br J Cancer 96 (Suppl): R40-R44, 2007.

4. Ryan BM, Robles AI and Harris CC: Genetic variation in microRNA networks: the implications for cancer research. Nat Rev Cancer 10: 389-402, 2010

5. Kasinski AL and Slack FJ: Epigenetics and genetics. microRNAs en route to the clinic: progress in validating and targeting microRNAs for cancer therapy. Nat Rev Cancer 11: 849-864, 2011.

6. Weber MJ: New human and mouse microRNA genes found by homology search. FEBS J 272: 59-73, 2005.

7. Gaur A, Jewell DA, Liang Y, et al: Characterization of microRNA expression levels and their biological correlates in human cancer cell lines. Cancer Res 67: 2456-2468, 2007.

8. Ruike Y, Ichimura A, Tsuchiya S, et al: Global correlation analysis for micro-RNA and mRNA expression profiles in human cell lines. J Hum Genet 53: 515-523, 2008.

9. Neville PJ, Conti DV, Krumroy LM, et al: Prostate cancer aggressiveness locus on chromosome segment 19q12-q13. 1 identified by linkage and allelic imbalance studies. Genes Chromosomes Cancer 36: 332-339, 2003.

10. Slager SL, Schaid DJ, Cunningham JM, et al: Confirmation of linkage of prostate cancer aggressiveness with chromosome 19q. Am J Hum Genet 72: 759-762, 2003.

11. Qu S, Yao Y, Shang C, et al: microRNA-330 is an oncogenic factor in glioblastoma cells by regulating SH3GL2 gene. PLoS One 7: e46010, 2012.

12. Lee KH, Chen YL, Yeh SD, et al: microRNA-330 acts as tumorsuppressor and induces apoptosis of prostate cancer cells through E2F1-mediated suppression of Akt phosphorylation. Oncogene 28: 3360-3370, 2009.
13. Chintharlapalli S, Papineni S, Ramaiah SK and Safe S: Betulinic acid inhibits prostate cancer growth through inhibition of specificity protein transcription factors. Cancer Res 67: 2816-2823, 2007.

14. Sankpal UT, Goodison S, Abdelrahim M and Basha R: Targeting Sp1 transcription factors in prostate cancer therapy. Med Chem 7: 518-525, 2011.

15. Pore N, Liu S, Shu HK, et al: $\mathrm{Sp} 1$ is involved in Akt-mediated induction of VEGF expression through an HIF-1-independent mechanism. Mol Biol Cell 15: 4841-4853, 2004.

16. Baranwal S and Alahari SK: miRNA control of tumor cell invasion and metastasis. Int J Cancer 126: 1283-1290, 2010.

17. Iorio MV and Croce CM: microRNAs in cancer: small molecules with a huge impact. J Clin Oncol 27: 5848-5856, 2009.

18. Watahiki A, Wang Y, Morris J, et al: microRNAs associated with metastatic prostate cancer. PLoS One 6: e24950, 2011.

19. Chiefari E, Brunetti A, Arturi F, et al: Increased expression of AP2 and Sp1 transcription factors in human thyroid tumors: a role in NIS expression regulation? BMC Cancer 2: 35, 2002.

20. Shi Q, Le X, Abbruzzese JL, et al: Constitutive Sp1 activity is essential for differential constitutive expression of vascular endothelial growth factor in human pancreatic adenocarcinoma. Cancer Res 61: 4143-4154, 2001.

21. Yao JC, Wang L, Wei D, et al: Association between expression of transcription factor Sp1 and increased vascular endothelia growth factor expression, advanced stage, and poor survival in patients with resected gastric cancer. Clin Cancer Res 10: 4109-4117, 2004

22. Zannetti A, Del Vecchio S, Carriero MV, et al: Coordinate up-regulation of Sp1 DNA-binding activity and urokinase receptor expression in breast carcinoma. Cancer Res 60: 1546-1551, 2000.

23. Chen JL, Attardi LD, Verrijzer CP, Yokomori K and Tjian R: Assembly of recombinant TFIID reveals differential coactivator requirements for distinct transcriptional activators. Cell 79: 93-105, 1994

24. Furukawa T and Tanese N: Assembly of partial TFIID complexes in mammalian cells reveals distinct activities associated with individual TATA box-binding protein-associated factors. J Biol Chem 275: 29847-29856, 2000.

25. Majello B, Napolitano G, De Luca P and Lania L: Recruitment of human TBP selectively activates RNA polymerase II TATA-dependent promoters. J Biol Chem 273: 16509-16516, 1998.

26. Sadovsky Y, Webb P, Lopez G, et al: Transcriptional activators differ in their responses to overexpression of TATA-box-binding protein. Mol Cell Biol 15: 1554-1563, 1995.

27. Murthy S, Ryan AJ and Carter AB: SP-1 regulation of MMP-9 expression requires Ser586 in the PEST domain. Biochem J 445: 229-236, 2012

28. Qin H, Sun Y and Benveniste EN: The transcription factors Sp1, $\mathrm{Sp} 3$, and AP-2 are required for constitutive matrix metalloproteinase-2 gene expression in astroglioma cells. J Biol Chem 274: 29130-29137, 1999

29. Wang $\mathrm{CH}$, Chang $\mathrm{HC}$ and Hung WC: p16 inhibits matrix metalloproteinase-2 expression via suppression of Sp1-mediated gene transcription. J Cell Physiol 208: 246-252, 2006. 